\title{
Perspective on the Response of Turbulent Pipe Flows to Strong Perturbations
}

\author{
Liuyang Ding ${ }^{1}$, Tyler Van Buren ${ }^{2}$, Ian E. Gunady ${ }^{1}(\mathbb{D})$ and Alexander J. Smits ${ }^{1, *(D)}$ \\ 1 Mechanical and Aerospace Engineering, Princeton University, Princeton, NJ 08544, USA; \\ liuyangd@princeton.edu (L.D.); igunady@princeton.edu (I.E.G.) \\ 2 Mechanical Engineering, University of Delaware, Newark, DE 19716, USA; vanburen@udel.edu \\ * Correspondence: asmits@princeton.edu
}

Citation: Ding, L.; Van Buren, T.; Gunady, I. and Smits, A.J. Perspective on the Response of Turbulent Pipe Flows to Strong Perturbations. Fluids 2021, 6, 208. https://doi.org/ 10.3390 / fluids 6060208

Academic Editor: Timothy Wei

Received: 2 April 2021

Accepted: 25 May 2021

Published: 4 June 2021

Publisher's Note: MDPI stays neutral with regard to jurisdictional claims in published maps and institutional affiliations.

Copyright: (c) 2021 by the authors. Licensee MDPI, Basel, Switzerland. This article is an open access article distributed under the terms and conditions of the Creative Commons Attribution (CC BY) license (https:// creativecommons.org/licenses/by/ $4.0 /)$.

\begin{abstract}
Pipe flow responds to strong perturbations in ways that are fundamentally different from the response exhibited by boundary layers undergoing a similar perturbation, primarily because of the confinement offered by the pipe wall, and the need to satisfy continuity. We review such differences by examining previous literature, with a particular focus on the response of pipe flow to three different kinds of disturbances: the abrupt change in surface condition from rough to smooth, the obstruction due to presence of a single square bar roughness elements of different sizes, and the flow downstream of a streamlined body-of-revolution placed on the centerline of the pipe. In each case, the initial response is strongly influenced by the pipe geometry, but far downstream all three flows display a common feature, which is the very slow, second-order recovery that can be explained using a model based on the Reynolds stress equations. Some future directions for research are also given.
\end{abstract}

Keywords: turbulence; pipe flow; perturbation; roughness

\section{Introduction}

This paper is part of the Special Issue honoring the contributions of W. W. Wilmarth to turbulence research. Bill Willmarth had a particular interest in turbulent boundary layers, especially the nature of the wall pressure fluctuations [1] and the scaling of the Reynolds stresses [2]. In addition, as noted by Blackwelder et al. [3], he posed provocative questions regarding turbulent wall-layer structures, like "What are the effects of parameters, such as wall-roughness, pressure-gradients, surface-curvature, and three-dimensionality on the structure elements? How do the evolutionary histories of structures change with the above parameters?"

With regard to the behavior of canonical flows, especially flat plate boundary layers and fully developed pipe and channel flows, the last 10 to 20 years has seen an intense research effort that has brought great progress. Through a combination of pioneering experiments and computations covering a wide Reynolds number range, our knowledge of such flows has improved greatly. It is now well accepted that classical scaling laws combined with Townsend's attached eddy hypothesis provide a fundamental framework for our present understanding. It has also become apparent that the effects of complexity can sometimes be included in this framework. For example, surface roughness can be incorporated simply as a change in the wall stress using Townsend's hypothesis [4], although the prediction of the wall stress as a function of the surface roughness is still a work in progress [5]. In addition, under certain conditions the effects of pressure gradient can be included in this framework by using a model for the detached eddies [6,7].

These findings, however, have been mostly restricted to either fully-developed flows or boundary layers that exhibit self-similarity, that is, they scale according to local variables, such as the local freestream velocity $U_{e}$, the friction velocity $u_{\tau}$, and the boundary layer thickness $\delta$. Here, $u_{\tau}=\sqrt{\tau_{w} / \rho}, \tau_{w}$ is the wall stress, and $\rho$ is the fluid density. 
Another class of flows, of particular interest here, as it was to Willmarth, concerns the response to strong perturbations. For example, an equilibrium boundary layer may experience a sudden onset of wall curvature or flow divergence, or meet with an abrupt change in surface roughness [8-11]. Such flows were reviewed by Reference [12] and have continued to draw interest since then [13-27].

Almost all of these investigations consider only boundary layers, while the response of pipe flow to strong perturbations has received only limited attention. The response of pipe flow is different, primarily because of the geometric confinement associated with internal flows and the constraints on the flow response imposed by continuity. In some respects, however, pipe flows are an ideal place to explore the response of a wall-bounded flow to strong perturbations, from an experimental and numerical viewpoint, because the upstream and far-downstream conditions are well known, and very well defined at any Reynolds number. In addition, pipe flows are of enormous practical importance [28], and in many applications experience a wide range of flow perturbations. Nonetheless, non-equilibrium pipe flows have not been explored to the same extent as their boundary layer counterparts, and the number of studies that have investigated the effects of strong perturbations on pipe flows is very small. Even if we include perturbed channel flows in the class of internal flows, the number does not grow much, and such studies are all relatively recent.

For example, Saito and Pullin [29] performed a high Reynolds number large eddy simulation (LES) study of turbulent flow in a long channel that experienced repeated transitions between smooth and rough surfaces. For a given transition, they found that the friction velocity first overshot and then undershot their equivalent equilibrium value before finally recovering over a stream-wise distance of order $10-30 h$, where $h$ is the channel half-height, depending on both roughness and Reynolds number. These observations are in line with the response of boundary layers to a change in surface roughness [30,31], but they noted the important role played by confinement due to the channel geometry. They used the example of a smooth-to-rough transition to illustrate this phenomenon. The velocity abruptly experiences a deficit near the wall as it first encounters the increase in surface roughness. To maintain continuity, this constitutes a loss of stream-wise mass flux near the wall that requires an equal increase of stream-wise mass flux farther away from the wall. Since the boundary layer has a semi-infinite vertical extent, such an increase in flux away from the wall is noted only as a slight increase in velocity. In a steady channel flow, however, velocity increases in the outer region are expected to be correspondingly more significant. This was indeed the case.

More recently, Ismail et al. [32-34] reported direct numerical simulations (DNS) of channel flow undergoing a step change from either a rib- or cube-roughened surface to a smooth wall. They estimated that the recovery distance in the outer flow was on the order of 50 channel half-heights, and different statistics were adjusting at dissimilar rates. Notably, the expansion of the flow after the step change produces strong advection effects and an adverse pressure gradient, which delay the emergence of the logarithmic law to an estimated distance of about $x / h=15-20$. The dominant momentum balance in the developing regime was between strong advection and turbulence fluxes.

Here, we discuss some recent experimental results that expand our understanding of strongly perturbed pipe flows [35-40]. Three cases are considered: the recovery from a step change in roughness from rough to smooth; the relaxation downstream of a single square bar roughness element of different heights; and the recovery of the wake downstream of a body-of-revolution placed on the centerline of the pipe. This summary of recent work is more in the service of assessing our present knowledge of perturbed pipe flows, rather than an original research contribution, and so we are particularly interested in drawing out the similarities observed in the more general flow response. We will see that this class of perturbed flows, especially their characteristically slow relaxation behavior, can be connected to a second-order flow model derived from the RANS equations. We begin 
by presenting brief summaries of the data for all three experiments, and then discuss the modeling effort and how it can be used to unify the observational experience.

\section{Flow Past a Step-Change in Wall Roughness}

Van Buren et al. [37] studied the propagation of disturbances in pipe flow following a rough-to-smooth step change in wall condition. The pipe radius $R$ was $19.1 \mathrm{~mm}$ with a bulk velocity of $3.45 \mathrm{~m} / \mathrm{s}$. This resulted in a diameter-based Reynolds number of $R e_{D}=1.31 \times 10^{5}$. The flow developed over sand-grain roughness, with an equivalent roughness of $k_{S}=415 \mu \mathrm{m}$, for a distance of 100 diameters before the surface abruptly changed to a smooth wall. The flow downstream of the step change was measured in cross-sectional planes-at $x=x_{s} / R=4.4,6.4,9.2,13.4,19.4,28.4 ., 41.2,60,80,100$, and 120 , where $x_{s}$ is measured from the start of the smooth pipe-using stereoscopic particle image velocimetry (SPIV), which gives all three components of velocity on a two-dimensional plane. Pressure measurements were also made at multiple locations downstream of the step using a freestanding manometer.

In a fully-developed pipe flow, the area- and time-averaged pressure gradient $\left\langle\frac{d P}{d x}\right\rangle$ balances the wall shear $\tau_{w}$ exactly, but, when the flow is in a state of development, the stream-wise gradient of the momentum flux $\left\langle\frac{d \rho \overline{\left(U_{x}+u_{x}\right)^{2}}}{d x}\right\rangle$ also contributes to the force balance. The notation is such that $U_{x}$ is the mean stream-wise velocity and $u_{x}$ is the fluctuating stream-wise velocity. Figure 1 shows the various contributions made by the momentum gradient, pressure gradient, and wall shear in the experiment as the flow responds to the step change. The development of the area-averaged turbulence kinetic energy $\langle K\rangle$ is also shown. This figure displays relative development, where the pressure gradient, skin friction, and turbulence kinetic energy are normalized by their values at the farthest downstream location, which was taken as an estimate of the fully relaxed state. For a short region downstream of the step change, the wall shear, momentum gradient, and pressure gradient are all lower than in the fully-developed smooth-wall case. These quantities recover relatively quickly, however, and, by $x=20$, they have approximately reached their fully-developed values. The turbulence kinetic energy level, however, displays small oscillations that persist much farther downstream. This oscillatory response of the flow statistics and its asymptotically slow relaxation, shown here by the turbulence kinetic energy, are unifying features of the three flows considered in this paper.

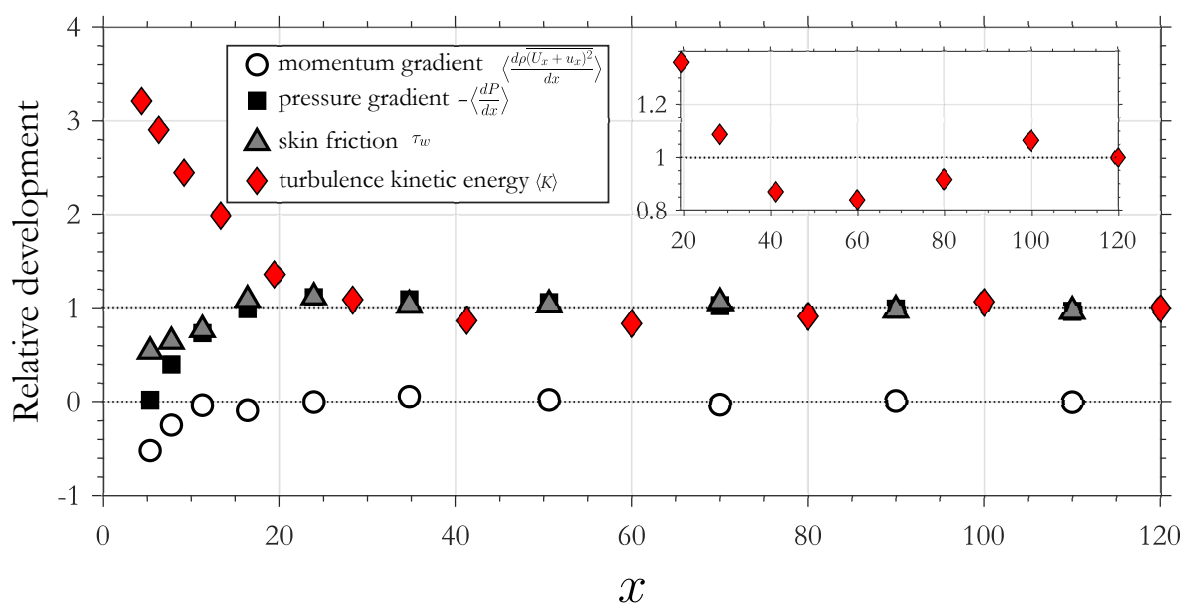

Figure 1. Stream-wise relative development of the pressure gradient, skin friction, stream-wise momentum gradient, and the cross-sectional area average of the turbulence kinetic energy. Here, $x=x_{s} / R$, where $x_{s}$ is measured from the start of the smooth pipe. (Reproduced with permission from [37], published by Cambridge University Press 2020).

As the flow moves from the rough pipe into the smooth one, the shear at the wall changes abruptly, and the subsequent acceleration at the wall must be balanced with a 
deceleration in the pipe center because of continuity. This can be most clearly seen via the downstream development of the mean stream-wise velocity profiles shown in Figure 2. The radial coordinate is $r / R$; we will also use the wall distance $y / R=1-r / R$. Note that all quantities are given non-dimensionally, normalized using the pipe radius $R$ and $u_{\tau 0}$, the friction velocity value expected to occur very far downstream of the step change.

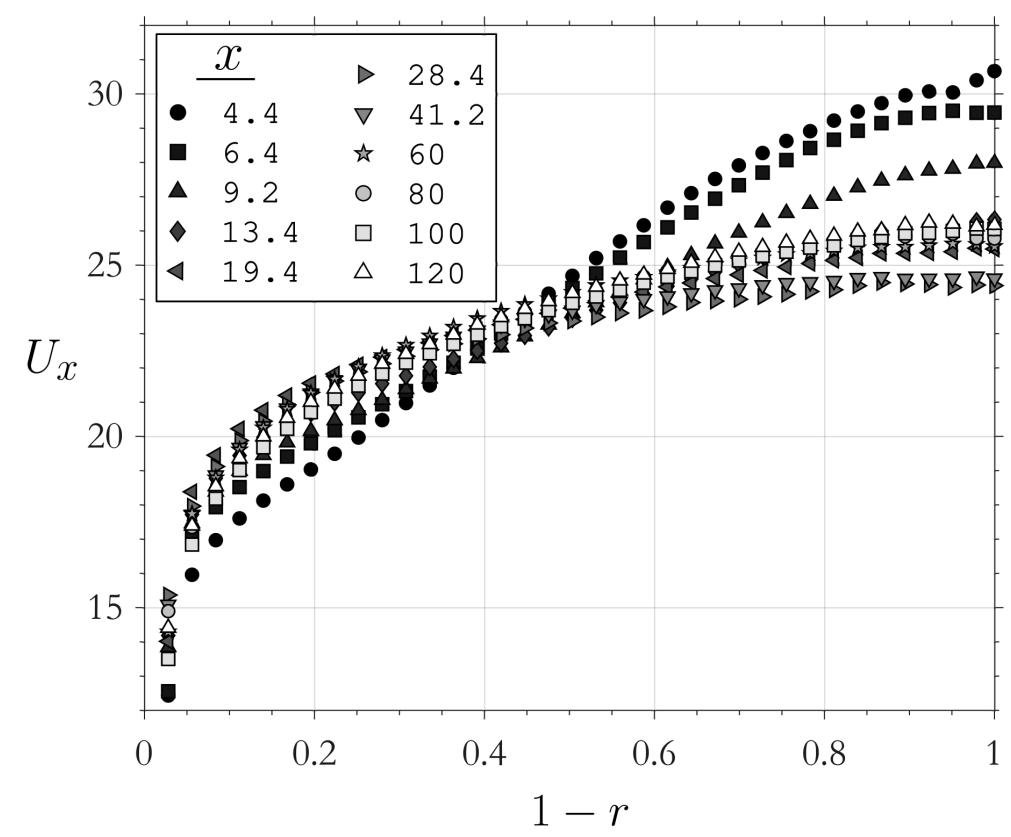

Figure 2. Downstream development of the mean stream-wise velocity profiles normalized by $u_{\tau 0}$ of turbulent pipe flow responding to a change in surface roughness. (Reproduced with permission from [37], published by Cambridge University Press 2020).

The flow can be analyzed using the Reynolds Averaged Navier-Stokes equations (RANS) for the stream-wise component (bearing in mind that we are considering a nonequilibrium flow)

$$
U_{x} \frac{\partial U_{x}}{\partial x}+U_{r} \frac{\partial U_{x}}{\partial r}=-\frac{\partial P}{\partial x}+\frac{1}{\operatorname{Re}_{\tau}} \nabla^{2} U_{x}-\frac{\partial \overline{u_{x}^{2}}}{\partial x}-\frac{1}{r} \frac{\partial \overline{r u_{x} u_{r}}}{\partial r} .
$$

The dominant acceleration is $U_{x} \frac{\partial U_{x}}{\partial x}$, and the data indicated it was balanced primarily by the wall-normal gradient of the Reynolds shear stress, $-\frac{1}{r} \frac{\partial \bar{r} u_{x} u_{r}}{\partial r}$. It is, therefore, useful to examine the Reynolds stress transport equation, which can be written as a balance of production, turbulent diffusion, pressure diffusion, pressure strain, viscous diffusion, and viscous dissipation.

According to the data shown in Figure 3, the turbulent diffusion terms are important very close to the step, but they quickly become negligible with distance downstream. Far downstream, the Reynolds stresses evolve through a simple balance between one of the production terms, $-\overline{u_{r}^{2}} \frac{\partial U_{x}}{\partial r}$, and the pressure strain term. The production acts as a source of the Reynolds stress $\overline{u_{x} u_{r}}$, and the pressure strain term works against anisotropy in the turbulence energy and is, thus, a sink of $\overline{u_{x} u_{r}}$. This observation is crucial to the development of the far-field recovery flow model given in Section 5. 
(a)
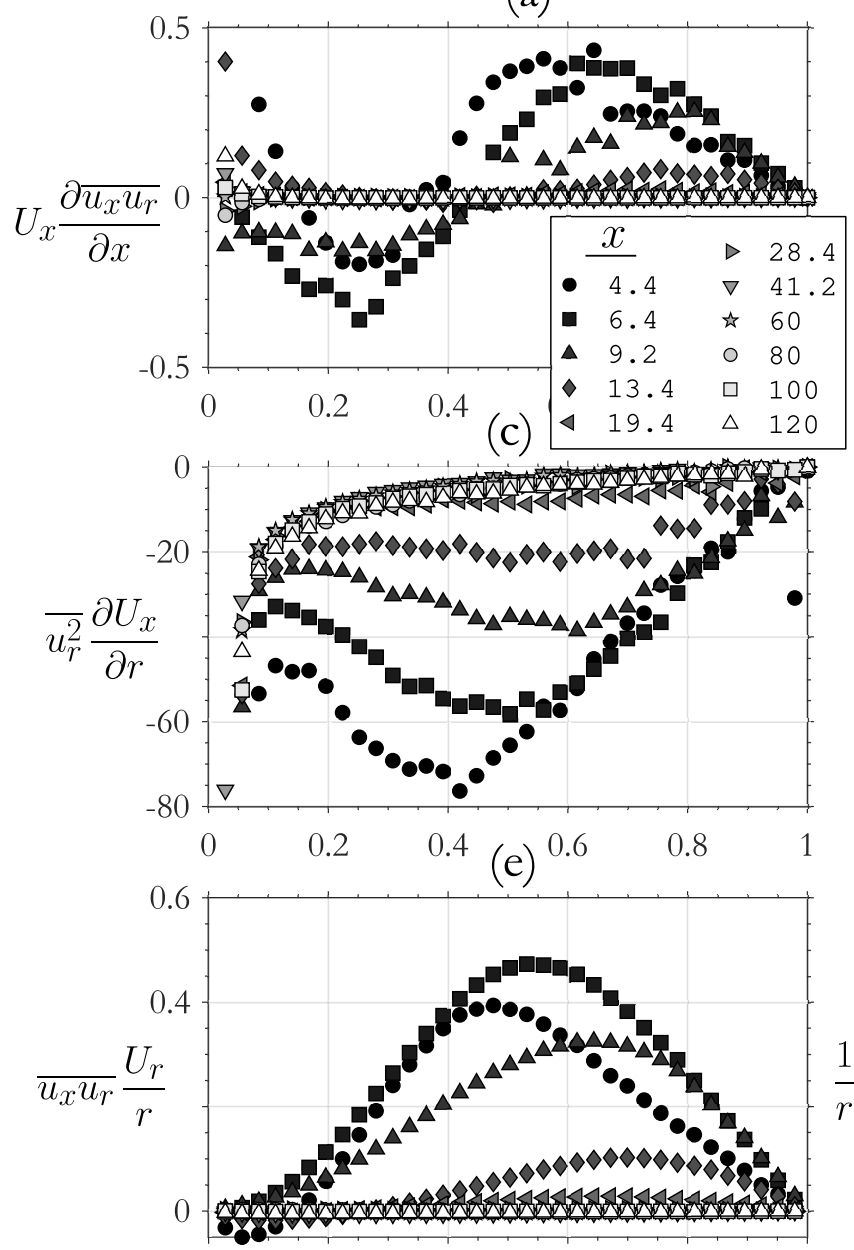

0

0.2

0.4 (g)

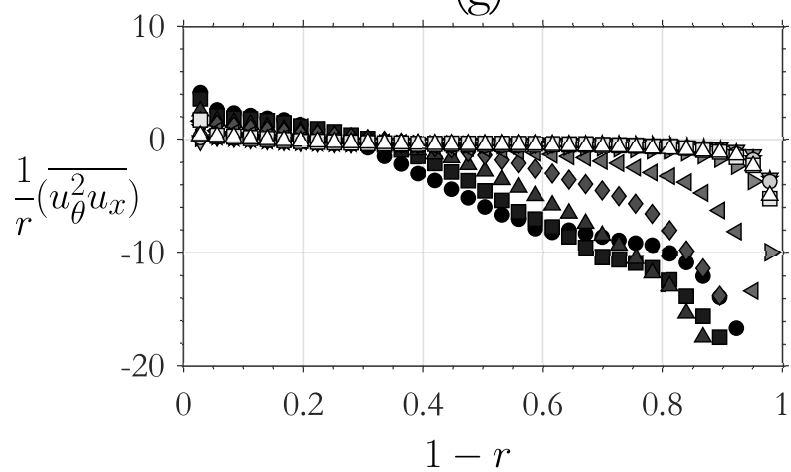

(b)
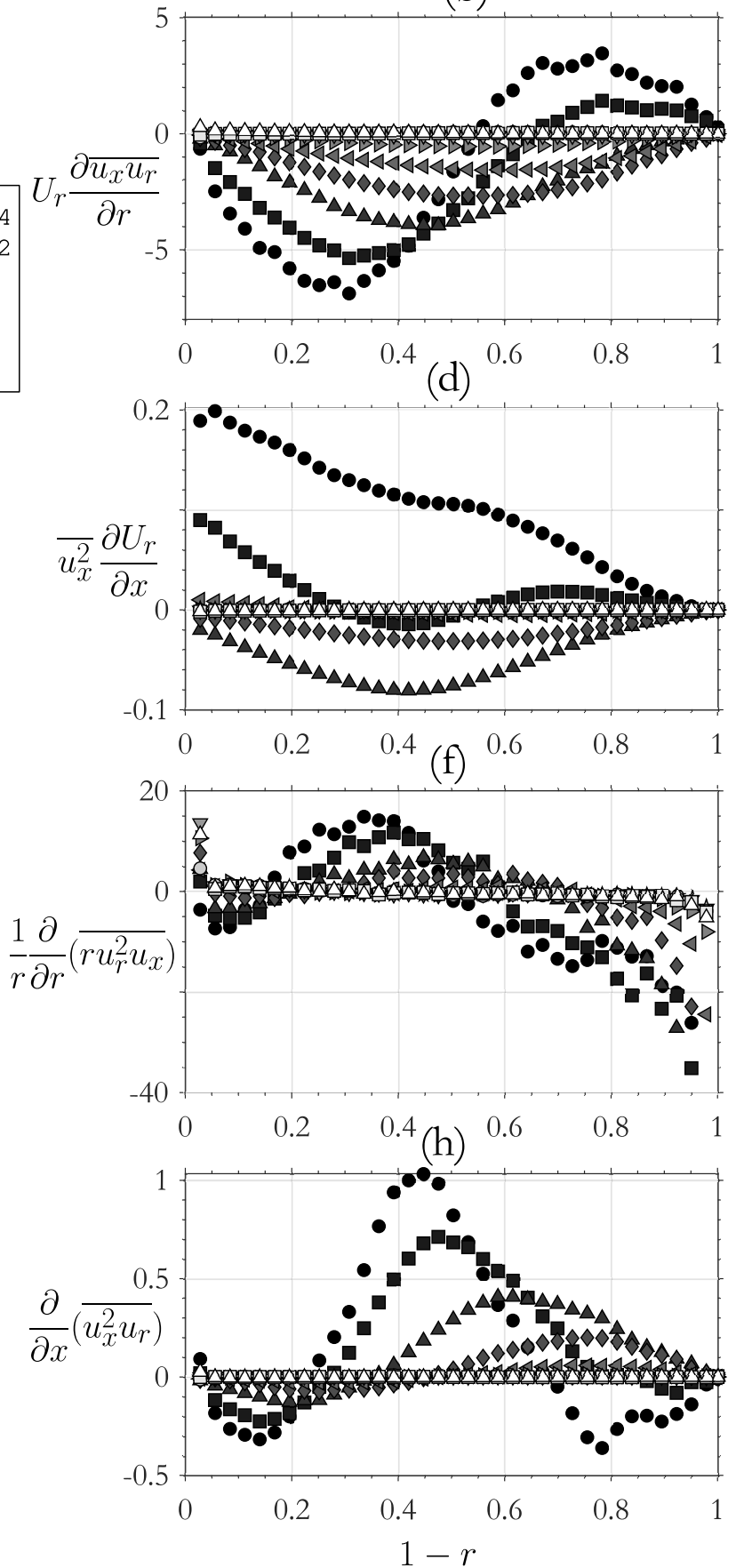

Figure 3. Downstream development of the terms in the transport equation for $\overline{u_{x} u_{r}}$ of turbulent pipe flow responding to a change in surface roughness. $(\mathbf{a}, \mathbf{b})$ mean advection; (c-e) production; $(\mathbf{f}-\mathbf{h})$ turbulent convection. (Reproduced with permission from [37], published by Cambridge University Press 2020).

Profiles of the different sources of turbulence kinetic energy: $\overline{u_{x}^{2}}, \overline{u_{r}^{2}}, \overline{u_{\theta}^{2}}$, and $\overline{u_{x} u_{r}}$, are presented for this experiment in Figure 4. These profiles indicated that, while some terms were larger than others, they all responded in a broadly similar to the change in wall condition. That is, for all the stresses, the peak of the disturbance is in the same radial location, the relative size of the peak is consistent, and the shape of the disturbance is approximately parabolic away from the centerline (these aspects were discussed in more detail by Reference [37]). In the simple model of the flow described in Section 5, therefore, it will be assumed that the disturbance takes the same shape for all fluctuation components. 


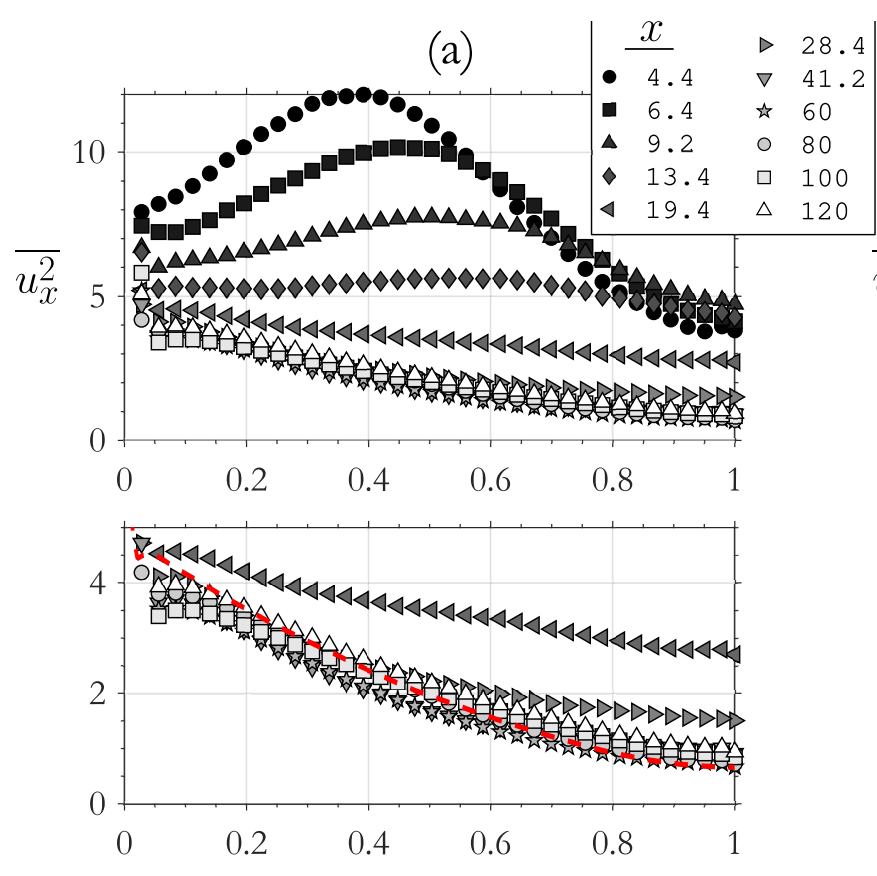

(c)
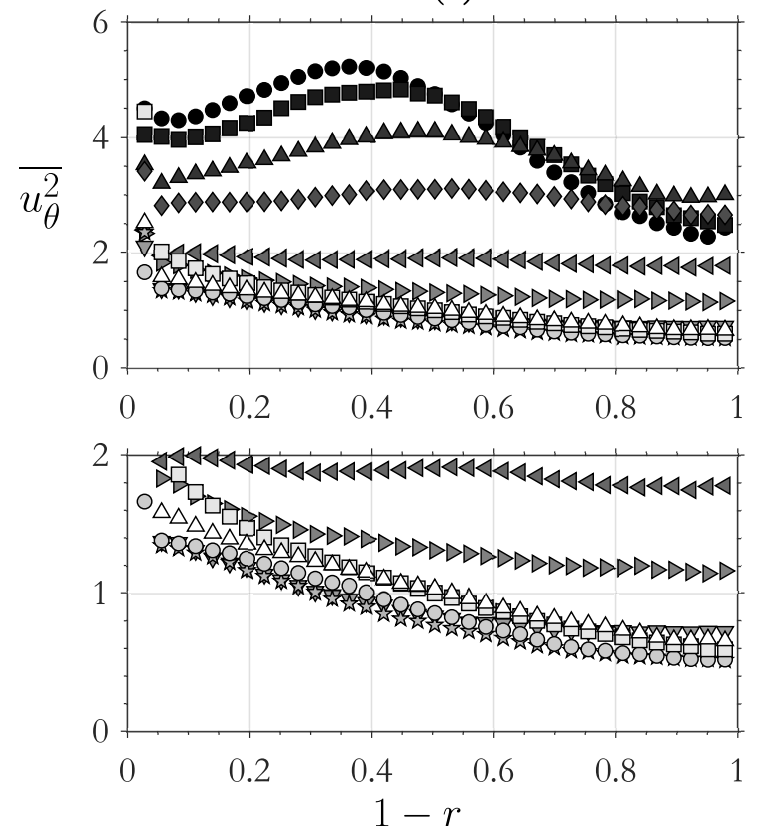

(b)
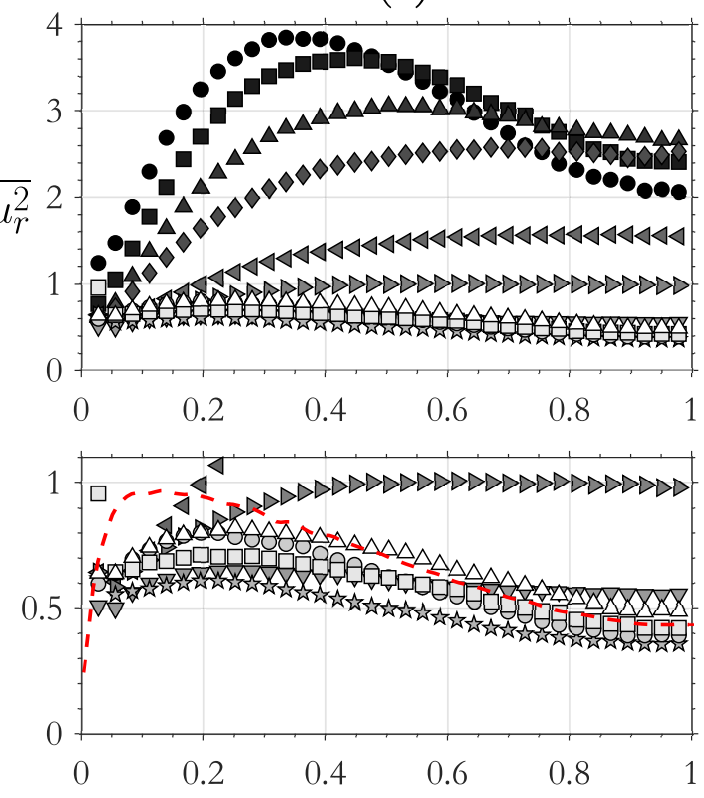

(d)
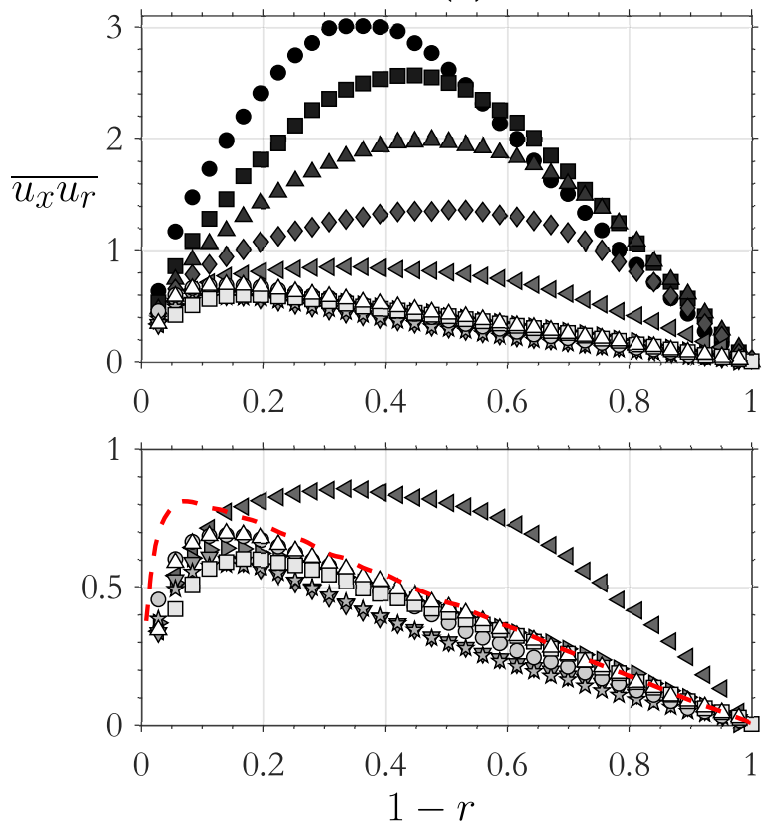

Figure 4. Downstream development of the Reynolds stresses in turbulent pipe flow responding to a change in surface roughness. The red dashed line marks the equilibrium profile. (Reproduced with permission from [37], published by Cambridge University Press 2020).

A particularly important finding is that the disturbances are evident very far downstream, exceeding the 120 pipe radii measurement domain. Additionally, the response was oscillatory, overshooting the eventual fully-developed condition in a second-order response.

To assess the influence of flow structures on the statistical response, Fourier decomposition and proper orthogonal decomposition may be applied in the azimuthal and radial directions, respectively. As has been found in the past [41,42], this approach can be used to identify large-scale motions in pipe turbulence. Van Buren et al. [37] found that the larger structures were the slowest to respond to the step-change in wall roughness, as is to be expected, and that they were primarily responsible for the second-order like response, which may not be as expected. 


\section{Flow Downstream of a Square Bar Roughness Element}

The next case we consider is the relaxation of turbulent pipe flow downstream of a square bar roughness element $[38,40]$. The experiment was conducted in the same recirculating pipe facility used by Van Buren et al. [37], and the perturbation was introduced axisymmetrically by mounting a square-cross-section ring on the pipe wall at about $100 \mathrm{D}$ downstream of the entrance to the pipe. Three bar heights, $h / R=0.04,0.1$ and 0.2 , were employed to vary the perturbation strength. The upstream flow was fully developed at a bulk Reynolds number $R e_{D}=165,000$, corresponding to a friction Reynolds number $R e_{\tau 0}=3760$. (The Reynolds number dependence of the flow for $h / R=0.1$ was recently examined using RANS modeling by Reference [43]). PIV measurements were performed in the axial-radial plane at distances up to $x / R=120(x$ is measured from the downstream side of the square bar).

The mean streamlines in the region close to the separation bubble are shown in Figure 5, together with the corresponding shear stress distributions. For all three bars, the flow is seen to separate at the leading edge of the bar, and then the mean dividing streamline rises a little above the location where $y=h$ before bending down toward the reattachment point. At the same time, the peak in shear stress moves slowly away from the wall.

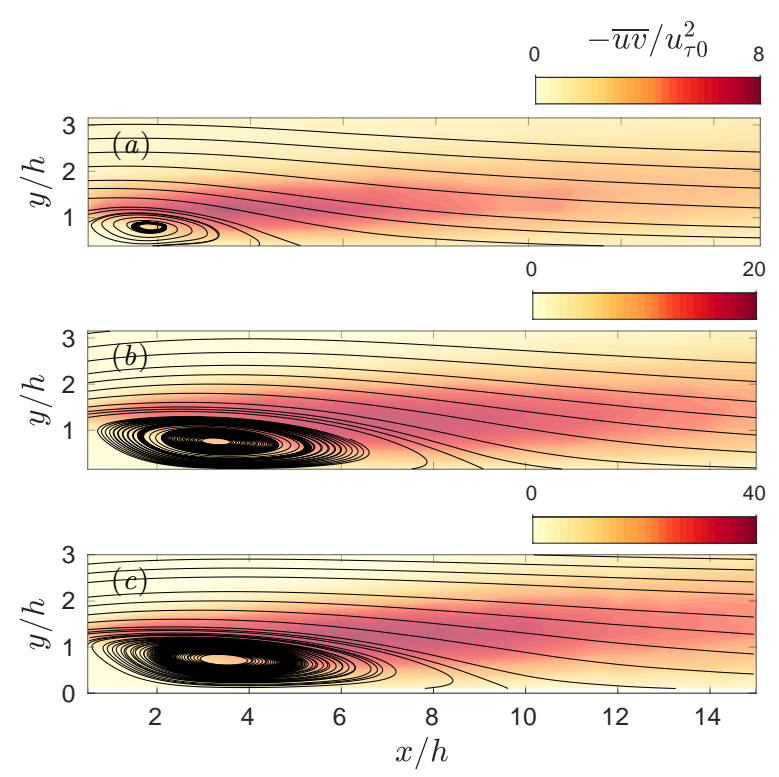

Figure 5. Mean streamlines near the separation bubble and flow reattachment distance. $(\mathbf{a}-\mathbf{c}): h / R=0.04$, $0.1,0.2$.

Ding and Smits [40] found that the recovery of the flow is characterized by different scaling laws and transport mechanisms in the near field and the far field, with their streamwise extents determined by $h / R$. In the near field $(x / h<100)$, the flow in the central region of the pipe first accelerates to maintain continuity and then gradually slows down, as shown in Figure 6a. The mean velocity profiles relax by pivoting: far from the wall the flow decelerates, while, near the wall, it speeds up. The mean momentum budget indicates that in the near field the near-wall acceleration is primarily attributed to momentum flux towards the wall, whereas the retardation in the central region is driven by adverse pressure gradients. It was also found that the mean pressure gradient recovers to its equilibrium value at about $x / h=50$, corresponding to $2 R, 5 R$, and $10 R$ for the small, medium, and large bars, respectively. In the far field, shown in Figure $6 \mathrm{~b}$, the mean flow relaxation slows down, but, by $x / R=120$, it has almost fully recovered. 

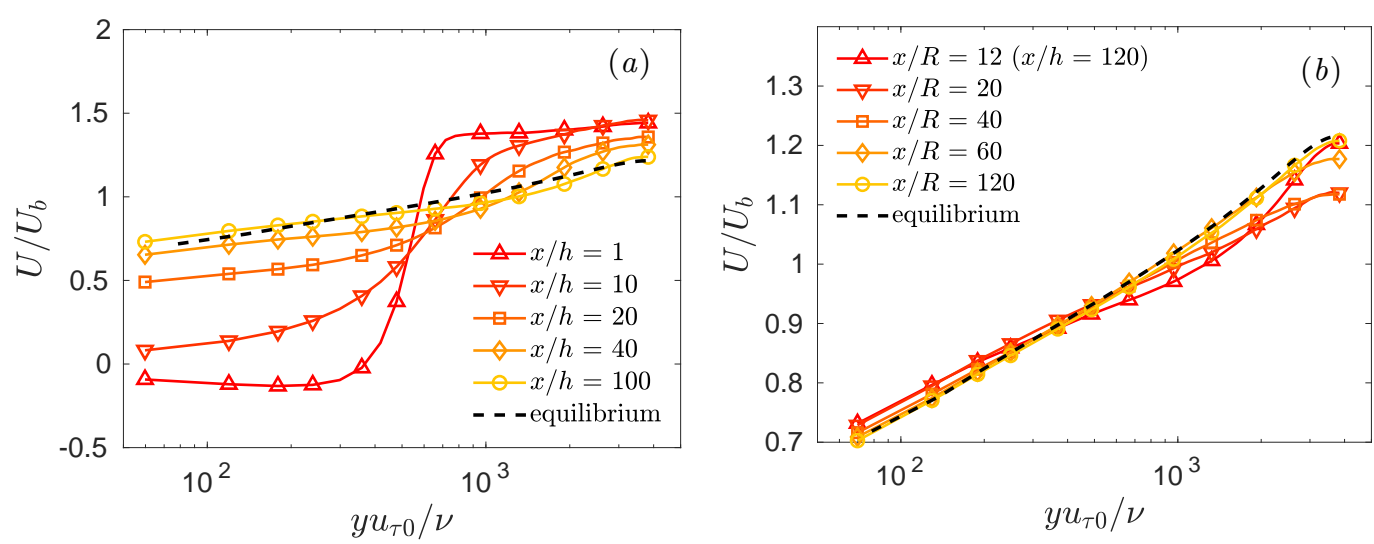

Figure 6. Distribution of $U$ at various locations downstream of a square bar roughness element for $h / R=0.1$. (a) Near field. (b) Far field.

The development of turbulent shear stress in the near field is presented in Figure 7a for the case where $h / R=0.1$. It is seen that the shear layer produced by the square bar is initially confined in a region close to $y / h=1$ (that is, $y / R=0.1$ ) before it starts to diffuse outwards. The diffusion or redistribution of turbulence is a result of turbulent convection in the wall-normal direction (i.e., the triple correlation term $\partial \overline{u v^{2}} / \partial y$ in the transport equation for $-\overline{u v}$ ). In the far field, shown in Figure $7 b$, the shear stress distribution for $y / R>0.1$ maintains an approximately similar shape as it decays, but it eventually falls below the equilibrium levels before recovering to its expected distribution by $x / R=120$.
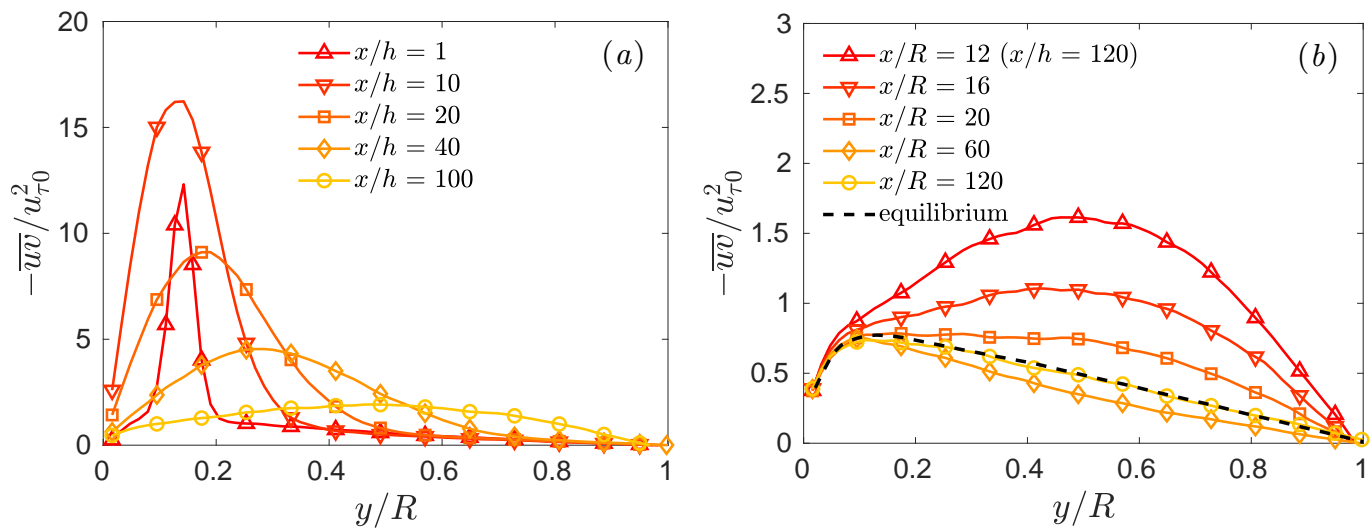

Figure 7. Distribution of $-\overline{u v}$ at various locations downstream of a square bar roughness element for $h / R=0.1$. (a) Near field. (b) Far field.

The scaling of the response is represented by the development of $y^{*}$ and $\Delta \tau^{*}$ as a function of $x / h$, as shown in Figure 8. Here, $\Delta \tau^{*}$ is the maximum excess shear stress with respect to the fully developed state in a given profile, and $y^{*}$ denotes the wall-normal location of $\Delta \tau^{*}$. Figure 8 a shows that downstream of $x=10 h, y^{*} / h$ moves towards the pipe centerline as a result of turbulent convection, and it closely follows a power law $(x / h)^{0.65}$. The power law variation holds up to the point where $y^{*} / R \approx 0.55$, which marks the end of the region where turbulent convection is important. This point corresponds to a stream-wise distance $x_{c}$, which Ding and Smits [40] called the convection length scale.

As to $\Delta \tau^{*}$, its amplitude scales with $h / R$, as is seen in Figure $8 \mathrm{~b}$ for both the growth and the decay region. The maximum $\Delta \tau^{*}$ occurs at approximately the reattachment distance $x_{R}$, and it then decays approximately as $x^{-1}$ up until the point where $x=x_{c}$.

We see that the redistribution of turbulence, in which the turbulent convection $\partial \overline{u v^{2}} / \partial y$ plays a central role, is a defining mechanism in the initial response of the flow. During the redistribution process, $\partial \overline{u v^{2}} / \partial y$ is negative near the wall and positive away from the wall, thus moving $-\overline{u v}$ towards the centerline (i.e., $y^{*}$ increases with $x$ ), as seen in 
Figure 7a. Given the axisymmetry of a pipe, $\partial \overline{u v^{2}} / \partial y$ is zero at both $y=R$ and $y=0$, so that the increase of $y^{*}$ ceases when $\partial u v^{2} / \partial y$ exhibits a more or less symmetric shape over $0<y / R<1$. The location where $y^{*}$ stops increasing is $x=x_{c}$, and it also marks the location where $\Delta \tau^{*}$ peels off from the -1 power law decay. It is worth emphasizing that $x_{c}$ scales inversely with $h / R$, that is, the convection process lasts longer for a smaller bar. However, when $h / R$ becomes sufficiently small, the magnitude of $\Delta \tau^{*}$ decreases to almost zero before $x=x_{c}$, as was observed for the small bar case $(h / R=0.04)$. For the larger bars, $x_{c} / h=100$ and $33\left(x_{c} / R=10\right.$ and 6.7$)$ for $h / R=0.1$ and 0.2 , respectively.
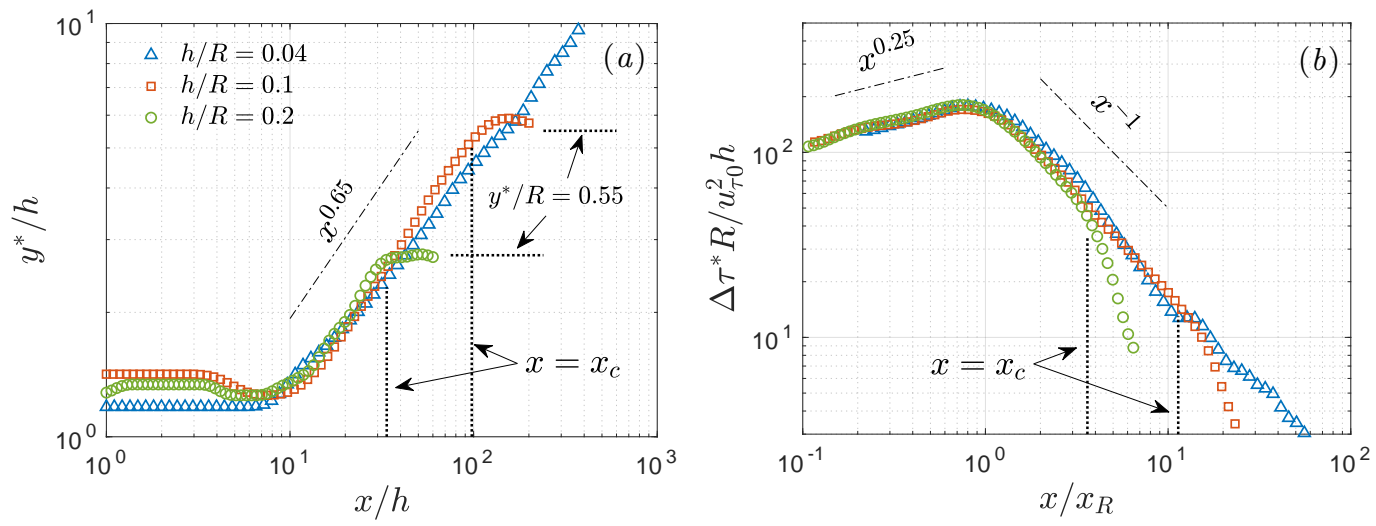

Figure 8. Development of (a) the wall-normal location of maximum shear stress, $y^{*}$, and (b) the maximum shear stress in a given profile, $\Delta \tau^{*} . y^{*}$ and $\Delta \tau^{*}$ are plotted against their respective near-field scaling, $x / h$ and $x / x_{R}$.

For the far-field development $\left(x>x_{c}\right)$, a few distinct features are observed in Figures $6 \mathrm{~b}$ and $7 \mathrm{~b}$. First, we see that the $-\overline{u v}$ profile maintains a roughly similar shape, while its peak value slowly decays, initially undershooting the equilibrium profile before finally reaching the equilibrium values by $x / R=120$. Second, the recovery is long-lasting with a stream-wise extent on the order of $100 R$, whereas the reattachment and convection occur within the initial 20R. In addition, both the mean flow and the turbulent shear stress exhibit an oscillatory recovery behavior. This is more obvious for the shear stress, which falls below the equilibrium profile before rising up again (Figure $7 \mathrm{~b}$ ).

Figure 9 presents the far-downstream development of the disturbance $x / x_{c}$. Here, $\Delta U, y$ is the maximum excess mean velocity gradient $\partial U / \partial y$ with respect to the fully developed state in a given profile, and similarly for $\Delta \tau_{b}$ where $\tau_{b}$ is the bulk (integrated) shear stress. Both $\Delta U_{, y}$ and $\Delta \tau_{b}$ display similarity in their stream-wise development when viewed against $x / x_{c}$, and $\Delta \tau_{b}$ lags behind $\partial \Delta U / \partial y$ by $\pi / 2$, that is, where $\Delta \tau_{b}$ crosses zero corresponds to the maximum $|\partial \Delta U / \partial y|$. The oscillation half-wavelength, as measured by the distance between the two zeros-crossings, is approximately $12.5 x_{c}$ for both $\partial \Delta U / \partial y$ and $\Delta \tau_{b}$. The similarity scale for the amplitudes of $\partial \Delta U / \partial y$ and $\Delta \tau_{b}$ was found to be simply $h / R$, as seen from the collapse of data in Figure 9. The picture of the far-field recovery then becomes clear-as the perturbation strength (as measured by $h / R$ ) increases, the mean flow statistics oscillate with a smaller wavelength $\left(\sim(h / R)^{-0.54}\right.$ in terms of $\left.R\right)$ and a larger amplitude $(\sim h / R)$. In addition, the oscillatory behavior is almost the same for the mean flow and the turbulence, except for a $\pi / 2$ phase difference. The above findings have made it clear that the scales governing the flow development transition from $h$ and $x_{R}$ in the near field to $x_{c}$ in the far field. 

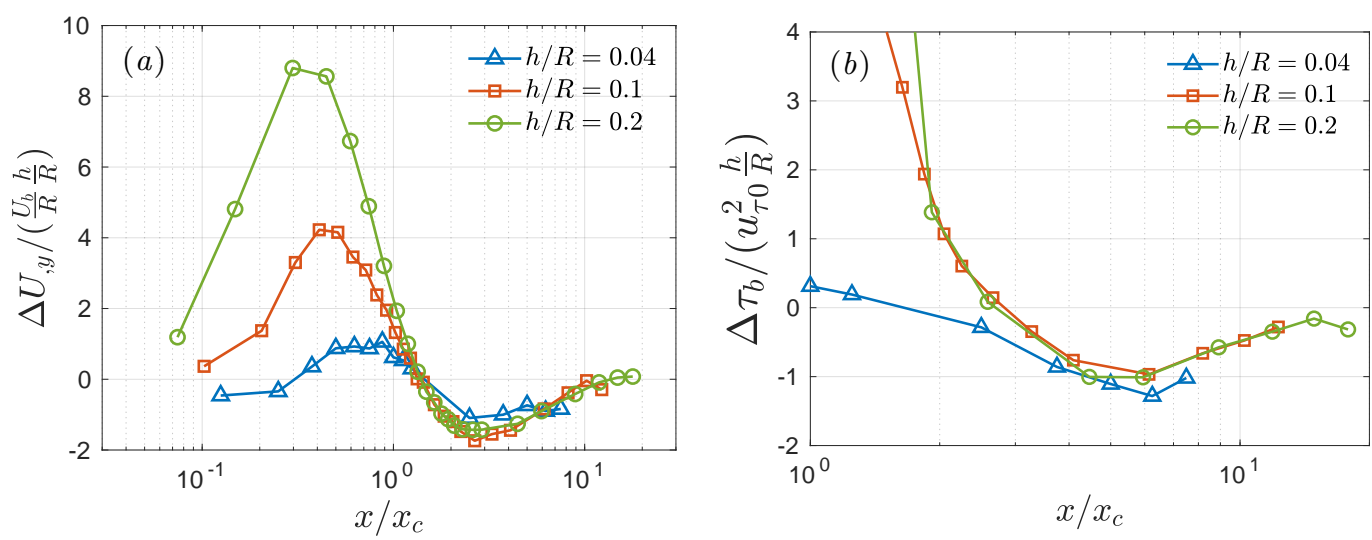

Figure 9. Development of (a) $\Delta U_{, y}$ and (b) $\Delta \tau_{b}$ in terms of $x / x_{c}$ in the final stage of relaxation. Here, $U, y$ denotes the wall-normal derivative of $U$, i.e., $U_{, y}=\partial U / \partial y$.

It was discussed in Reference [40] that the far-downstream recovery is driven by the interaction between the mean flow and the turbulent shear stress, and the oscillatory behavior results from the asynchronous recovery between the two quantities. The oscillation amplitude decays for both quantities, and the final recovery is achieved when the oscillation amplitude becomes sufficiently small. The damped oscillatory recovery has its roots in the transport equations and is a common behavior for relaxing wall-bounded flows $[9,32,37]$.

\section{Flow Downstream of a Streamlined Body of Revolution}

Both the step roughness change and the single square bar roughness element are perturbations in wall conditions. Here, we discuss a different test case where the pipe flow is perturbed by a streamlined body of revolution placed symmetrically on the centerline, as shown in Figure 10. As in the other cases, the upstream unperturbed flow was fully developed with a bulk Reynolds number of 165,000. The body consisted of three sections-a prolate spheroidal bow section, a cylindrical mid-body region, and a stern section with a sharp tail designed to avoid separation. The body affects the flow in a complex manner involving spatially-developing pressure gradients and streamline curvature and divergence/convergence. Three body diameters were investigated, with blockage ratios of $1 / 9$, $2 / 9$, and $1 / 3$. Planar PIV data were collected in the axial-radial plane over the entire body length, as well as in the near and far wakes. Here, we focus on the flow relaxation in the wake of the body with a blockage ratio of $1 / 3$. Further detail of the body geometry, as well as the flow development in the bow and mid-body regions, can be found in Reference [44].

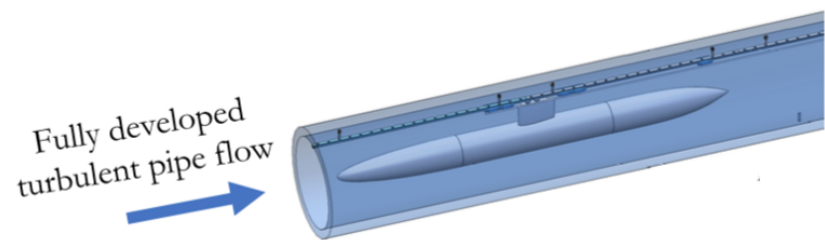

Figure 10. Body of revolution mounted on centerline of pipe. The focus is on the far-downstream recovery of the wake flow.

The mean momentum budget indicates that immediately downstream of the body the mean flow development is driven by both the pressure gradient $(-\partial P / \partial x)$ and the turbulent shear stress $(-\partial \overline{u v} / \partial y)$. The mean pressure gradient then recovers quickly in a few radii so that, farther downstream, the variation of $U$ is primarily due to momentum flux. The evolution of momentum flux, as characterized by $-\overline{u v}$, is governed by the interaction between the mean flow and Reynolds stress, which is a slow and oscillatory process, similar to the relaxation downstream of the step roughness change and the square bar roughness element. 
Figure 11 presents the evolution of the mean axial velocity in the wake, where $x$ is measured from the stern, $y$ is the wall-normal coordinate, and $R$ is the pipe radius. The mean velocity distribution in the wake can be viewed in terms of three regions-a layer near the pipe wall, a velocity defect region in the central part of the flow (the true wake, approximately defined by $y / R>0.6$ in this example), and a high-speed region centered at about $y / R=0.4$ that forms as a result of the flow acceleration due to blockage. The velocity in the wake undergoes an initial rapid recovery followed by a much slow development farther downstream. As shown in the figure, the velocity at the centerline accelerates from $15 \%$ to $80 \%$ of $U_{c 0}$ over the initial 10 radii (where $U_{c 0} \approx 1.2 U_{b}$ is the equilibrium centerline velocity), and then increases slowly to about $0.95 U_{c 0}$ by $x / R=34$. To maintain continuity, the acceleration near the centerline is accompanied by the deceleration seen for $0.15<y / R<0.6$, forming a pivot point near $y / R=0.6$. This pivoting behavior was also observed in the relaxation downstream of the square bar perturbation (see above) and is associated with momentum transfer by turbulent mixing [40]. As to the near-wall layer, it speeds up exiting the stern region, and it develops an overshoot above the fully-developed profile at $x / R=10$. Farther downstream at $x / R=34$, the mean velocity distribution over $0<y / R<1$ nearly coincides with the equilibrium profile, except for a slight defect near the centerline.

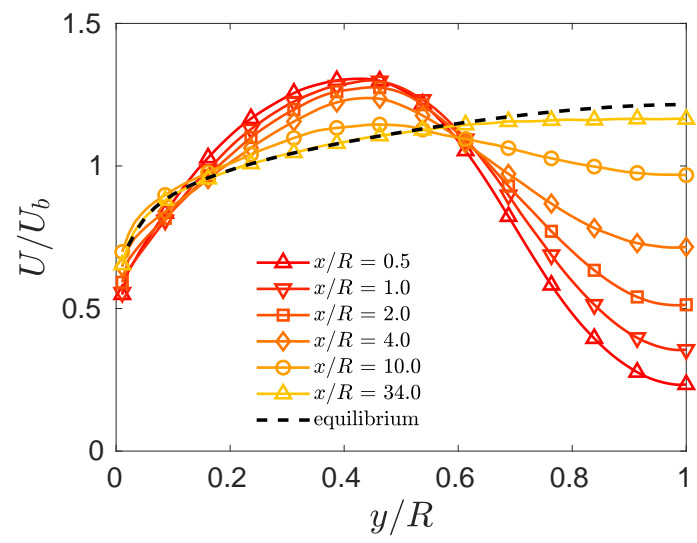

Figure 11. Development of the mean axial velocity in the wake of the streamlined body of revolution (body blockage ratio $=1 / 3$ ).

The relaxation of turbulent shear stress is shown in Figure 12a. Intense turbulent mixing is seen near both the pipe wall and the centerline. Near the pipe wall, the peak of $-\overline{u v}$ occurs at $y / R \approx 0.1$ immediately downstream of the body and then moves to $y / R \approx 0.2$ followed by a decay towards the equilibrium profile. This redistribution and decay process for the turbulent shear stress was also observed in the earlier examples for the step change in roughness and the square bar element where the perturbation produces excess turbulence near the wall $[37,40]$. Away from the wall in the region of negative velocity gradient $(0.4<y / R<1),-\overline{u v}$ changes sign, implying a mean momentum exchange in the opposite direction. The overall relaxation of $-\overline{u v}$ occurs at a high rate initially and then drastically slows down as it approaches the equilibrium profile, much like that observed in other perturbed wall turbulence flows. The redistribution and decay is also observed for the evolution of $\overline{u^{2}}$ in Figure $12 \mathrm{~b}$. The second-order response is again evident-an undershoot below the equilibrium profile is seen at $x / R=18$ and 34 . The fact that the distribution of $\overline{u^{2}}$ changes very little over $18<x / R<34$ suggests that the second-order response has a rather long wavelength and the recovery overall is long-lasting, a behavior that is again displayed by the step roughness change and the single square bar roughness element. Additional measureemtns farther downstream will be needed to investigate this behavior more fully. 

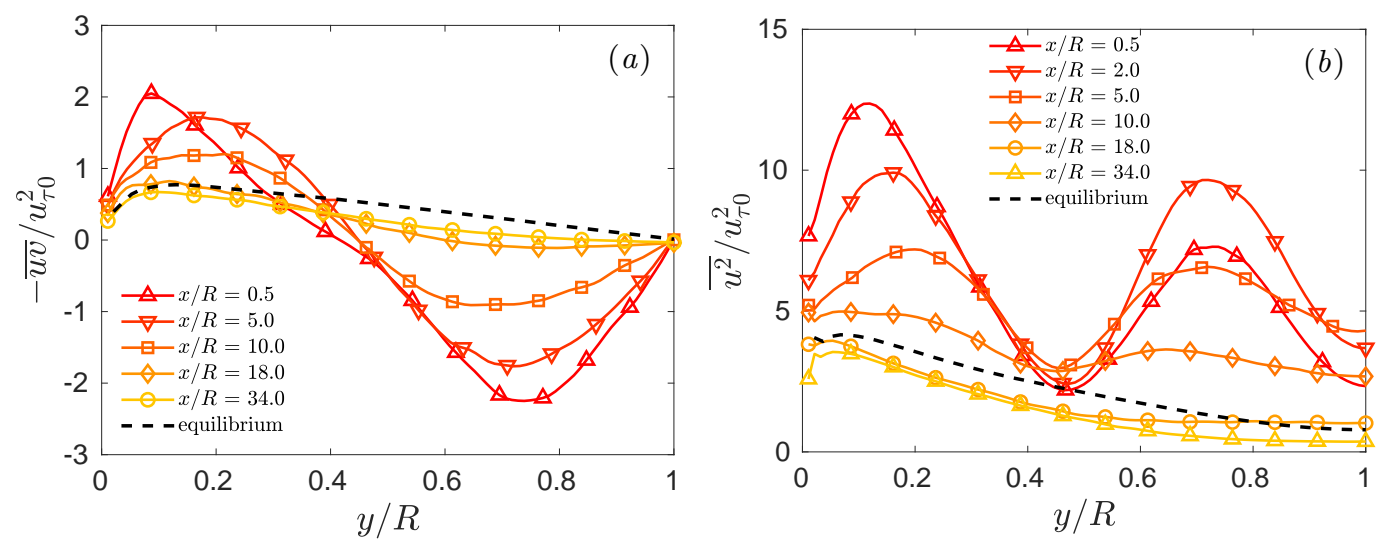

Figure 12. Development of (a) the Reynolds shear stress and (b) the axial turbulence intensity in the wake of the streamlined body of revolution (body blockage ratio $=1 / 3$ ).

\section{Modeling Non-Equilibrium Wall-Bounded Turbulence}

For all three examples discussed here, for the region beyond where turbulent convection/diffusion is important, we have a balance between production and pressure strain. In addition, in this region the disturbance profile (the measure of how far the stresses are away from their equilibrium values) maintains an approximately similar shape with distance downstream, and it can be characterized by a single variable, its peak height. This leads us to consider a model for the far-field recovery in terms of the RANS equations, particularly the shear stress transport equation.

Non-equilibrium turbulence has been successfully modeled in the past, with limitations. Both Reference $[9,37]$ developed a model for perturbed wall-bounded turbulence where the shape of the disturbance is assumed. The models describe the outer flow response where the large-scale motions with long time scales are key to understanding the long-lasting second-order response. Below is a summary of this modeling strategy, most recently used by Reference [37,40].

The model is based on two equations. First, the stream-wise RANS equation, differentiated by the wall-normal coordinate $r$ and re-organized using continuity,

$$
\frac{\mathrm{D}}{\mathrm{D} t}\left(\frac{\partial U_{x}}{\partial r}\right)-\frac{U_{r}}{r} \frac{\partial U_{x}}{\partial r}=\frac{\tau}{r^{2}}-\frac{1}{r} \frac{\partial \tau}{\partial r}-\frac{\partial^{2} \tau}{\partial r^{2}}
$$

Note here that $\tau=\overline{u_{x} u_{r}}$. Stream-wise gradients outside of the material derivative are neglected because the flow development itself is governed by wall-normal gradients. The second equation is the transport of $\tau$ :

$$
\frac{\mathrm{D} \tau}{\mathrm{D} t}=-\overline{u_{r}^{2}} \frac{\partial U_{x}}{\partial r}+\overline{p\left(\frac{\partial u_{x}}{\partial r}+\frac{\partial u_{r}}{\partial x}\right)}
$$

where it is assumed that the turbulent and pressure diffusion in the outer layer are negligible (supported by Reference [45]). Therefore, the transport of $\tau$ is governed by the competing contributions from production and pressure strain. The pressure strain terms are then broken into the "slow" and "fast" components [46] and then modeled following References [47,48].

These two main equations are then perturbed as follows:

$$
\begin{array}{lll}
U_{x}=U_{x, 0}+\Delta U_{x} ; & U_{r}=U_{r, 0}+\Delta U_{r} ; & \tau=\tau_{0}+\Delta \tau ; \\
\overline{u_{x}^{2}}=\overline{u_{x, 0}^{2}}+C_{x} \Delta \tau ; & \overline{u_{r}^{2}}=\overline{u_{r, 0}^{2}}+C_{r} \Delta \tau ; \quad \overline{u_{\theta}^{2}}=\overline{u_{\theta, 0}^{2}}+C_{\theta} \Delta \tau:,
\end{array}
$$

where subscript 0 denotes the downstream fully-developed case, and $\Delta$ refers to perturbation quantities. Notice here that each component of the mean flow gets its own perturbation, but all of the stresses are assigned the same perturbation profile $\Delta \tau$ with a unique scaling co- 
efficient $\left(C_{x}, C_{r}, C_{\theta}\right)$. The disturbance function is then further simplified by being separated into functions of the stream-wise and wall-normal coordinates $\Delta \tau(x, r)=\mathcal{T}(x) f(r)$.

After re-organization and simplification (for details see Reference [37]), the two main equations are used to construct the general form of the final model equation, given by

$$
\frac{\partial^{2} \mathrm{X}}{\partial x^{2}}+A \frac{\partial \mathrm{X}}{\partial x}+B \mathrm{X}=0
$$

where $\mathrm{X} \equiv \partial \Delta U_{x} / \partial r$. Here, the coefficient $A$ depends on the shape of the fully-developed velocity profiles and the disturbance amplitude, and coefficient $B$ depends on the shape of the disturbance itself. At a given radial location or integrating the equation over the pipe cross-section, this equation becomes a solvable ordinary second-order differential equation that takes the form of a damped harmonic oscillator, and so the model can give decayed oscillations in response, as we have seen by experiment.

This model was then validated against data from the case with a step change in surface roughness, as shown in Figure 13. As we can see, far downstream from the original disturbance, the model reproduces the nature of the oscillatory response (in both amplitude and frequency), as well as the longevity of the disturbance itself.

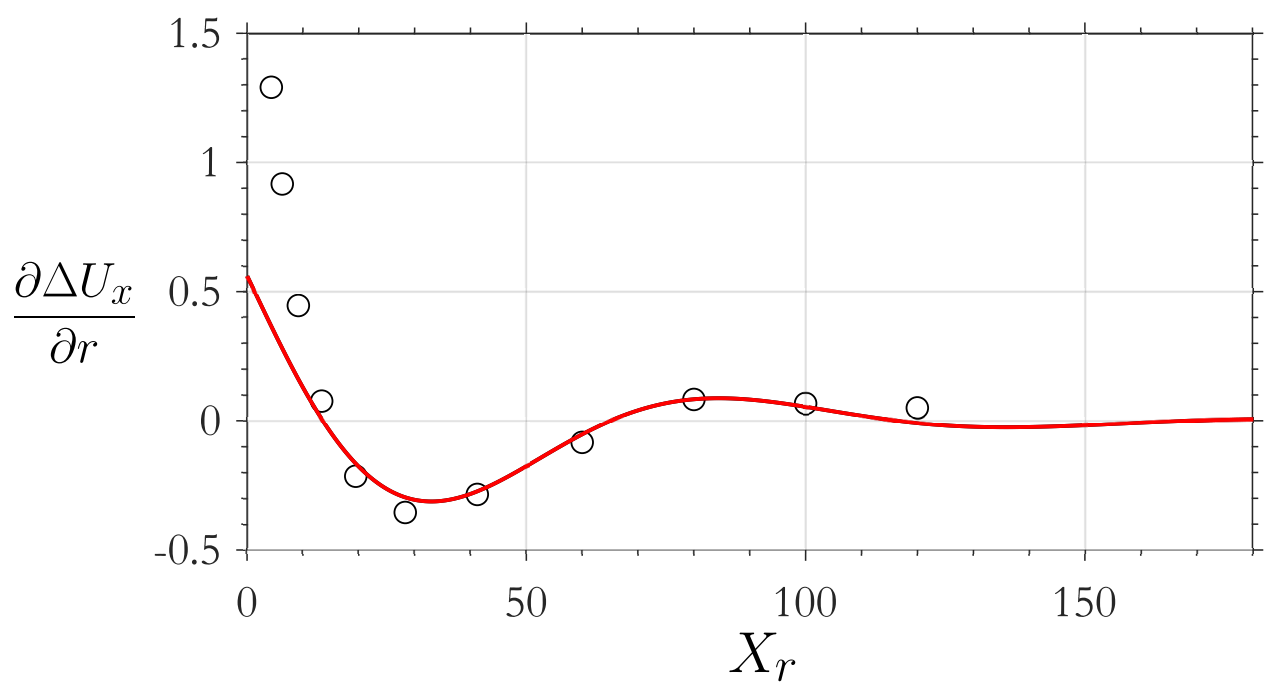

Figure 13. Comparison of the non-equilibrium turbulence model to turbulent pipe flow subject to a change in surface roughness. $X_{r}=x / R$ is the normalized stream-wise distance downstream of the rough-to-smooth step. (Reproduced with permission from [37], published by Cambridge University Press 2020).

Ding and Smits [40] further explored this model and tested it against the case of the square bar roughness element. They showed that, within the framework of linearized RANS models, the equation governing the transport of $-\overline{u v}$ has exactly the same form as the one governing $\partial U / \partial y$ (Equation (5)). It implies that $\partial U / \partial y$ and $-\overline{u v}$ follow the same damped oscillatory behavior, except for a phase difference. This is also in agreement with the results in the step roughness case-both the mean shear and the turbulent kinetic energy oscillate with a half-wavelength of approximately $55 R$, as seen from Figures 1 and 13 . Note that, since the coefficients $A$ and $B$ in Equation (5) are flow-dependent, the wavelengths for the step roughness change and the square bar roughness element are not the same (a wavelength of $120 R$ was found for $h / R=0.1$ in the square bar case). Moreover, Ding and Smits [40] showed that the linearized model was insufficient to capture the similarity in terms of the convection length scale (Figure 9).

The model has not yet been applied to the recovery of the wake downstream of the body of revolution. This flow exhibits most the features seen in the response to perturbations that originate near the wall, especially farther into the wake, and, qualitatively, it 
looks like a good candidate for a similar RANS-based approach. Quantitative analysis, however, will require additional data to document the far downstream recovery, since the flow has not yet fully recovered by the last downstream station at $x / R=34$.

\section{Opportunities for Future Research}

When turbulent pipe flow is perturbed, the confined geometry and the need to satisfy continuity lead to an immediate response in the mean velocity over the entire cross-section of the pipe. This is in contrast to boundary layers subjected to similar perturbations, in which the mean flow response is diminishing at locations having sufficient wall-normal separations from the perturbation. Associated with the mean velocity response in a pipe is the pressure response that is also immediate and global. The pressure response arises in the form of favorable or adverse pressure gradients, which are important in the near-field recovery of the mean velocity. However, the stream-wise extent over which pressure is important is relatively short, with a time scale similar to that of the perturbation itself, and the pressure gradient recovers much more quickly as compared to the mean velocity and higher order moments. In addition, the response of the turbulence (i.e., Reynolds stresses and other high-order moments) may be decoupled from the mean velocity, depending on the perturbation time scale. This is the scope of rapid distortion theory, and the decoupling elevates the complexity of the downstream flow relaxation.

It is clear that turbulent convection in the wall-normal direction plays an important role in the relaxation process. When intense turbulence is produced or excess turbulence is present near the location of the perturbation, it is diffused by turbulent convection and spreads out in the wall-normal direction. The convection of shear stress acts to shape the mean velocity distribution, and it sets a stream-wise length scale for the flow development in the far field of the perturbation. In the case where the new wall condition persistently creates excess turbulence, such as the flow downstream of a stabilizing curvature or a smooth-to-rough change, turbulent convection moves the excess turbulence away from the wall at a rate that dictates the growth of an internal layer. As to the comparison between boundary layers and pipe flow, the convection process continues to the edge of a boundary layer, while, in a pipe, the convection may stop before it reaches the centerline, as seen downstream of the square bar for the convection of $-\overline{u v}$. This difference is attributed to the axisymmetry of a pipe that impose a condition of zero net convection $\left(\partial \overline{u v^{2}} / \partial y\right)$ at the centerline.

Most interestingly, the far-downstream recovery exhibits a common behavior that is both slow and oscillatory. Such behavior has its roots in the asynchronous development of the mean velocity and the turbulent shear stress. A model based on the equations describing the transport of $U$ and $-\overline{u v}$ was described. It was based on the ideas originally presented by Reference [9] in the context of a boundary layer recovering from an impulse in surface curvature, and it provides insights into the physics of the recovery. In particular, it is adept at reproducing the damped second-order response, and it appears to provide a reasonable basis for modeling (and understanding) different types of non-equilibrium wall-bounded turbulence beyond pipe flow.

To further explore the potential of pipe flow as a forum for examining the response to a range of perturbations, a number of possibilities can be suggested. In this respect, the effects of Reynolds number on the response can be investigated rather cleanly, in the sense that the bulk Reynolds number can easily be varied without changing the history of the upstream flow. This aspect of pipe flow could be useful in, for example, determining more precisely the effects of Reynolds number on separation, which is a difficult to do in the context of boundary layers where the development with downstream distance always needs to be taken into account. In addition, it is straightforward to expand the suite of perturbations that can be introduced, such as flow curvature, flow convergence and divergence, surface heat transfer, flow suction and blowing, and so on. All these flows have their counterpart in industrial applications, as well as provide new challenges to our understanding and the development of turbulence models. 
Author Contributions: Writing—original draft preparation, review, and editing A.J.S., L.D., T.V.B., L.D., and I.E.G. acquired and analyzed the data for the body of revolution. All authors have read and agreed to the published version of the manuscript.

Funding: This research was funded by ONR grant number N000141712309 (Program Manger Peter Chang). I.E.G. was supported by the National Defense Science \& Engineering (NDSEG) Fellowship Program.

Conflicts of Interest: The authors declare no conflict of interest.

\author{
Abbreviations \\ The following abbreviations are used in this manuscript: \\ MDPI Multidisciplinary Digital Publishing Institute \\ DOAJ Directory of open access journals \\ TLA Three letter acronym \\ LD Linear dichroism
}

\title{
References
}

1. Willmarth, W.W. Pressure fluctuations beneath turbulent boundary layers. Annu. Rev. Fluid Mech. 1975, 7, 13-36.

2. Wei, T.; Willmarth, W.W. Reynolds-number effects on the structure of a turbulent channel flow. J. Fluid Mech. 1989, 204, 57-95.

3. Blackwelder, R.F.; Panton, R.L.; Wallace, J.M. Questions from the Willmarth Workshop on New Directions in Research on Turbulent Wall-Layer Structures; NASA STI/Recon Technical Report N; NASA: Washington, DC, USA, 1989.

4. Allen, J.J.; Shockling, M.A.; Smits, A.J. Evaluation of a universal transitional resistance diagram for pipes with honed surfaces. Phys. Fluids 2005, 17, 121702. [CrossRef]

5. Chung, D.; Hutchins, N.; Schultz, M.P.; Flack, K.A. Predicting the drag of rough surfaces. Annu. Rev. Fluid Mech. 2021, 53, 439-471. [CrossRef]

6. Perry, A.E.; Marusic, I. A wall-wake model for the turbulence structure of boundary layers. Part 1. Extension of the attached eddy hypothesis. J. Fluid Mech. 1995, 298, 361-388. [CrossRef]

7. Marusic, I.; Perry, A.E. A wall wake model for the turbulent structure of boundary layers. Part 2. Further experimental support. J. Fluid Mech. 1995, 298, 389-407. [CrossRef]

8. Mulhearn, P.J. A wind-tunnel boundary-layer study of the effects of a surface roughness change: Rough to smooth. Bound.-Layer Meteorol. 1978, 15, 3-30. [CrossRef]

9. Smits, A.J.; Young, S.T.B.; Bradshaw, P. The effect of short regions of high surface curvature on turbulent boundary layers. J. Fluid Mech. 1979, 94, 209-242. [CrossRef]

10. Smits, A.J.; Eaton, J.A.; Bradshaw, P. The response of a turbulent boundary layer to lateral divergence. J. Fluid Mech. 1979, 94, 243-268. [CrossRef]

11. Andreopoulos, J.; Wood, D.H. The response of a turbulent boundary layer to a short length of surface roughness. J. Fluid Mech. 1982, 118, 143-164. [CrossRef]

12. Smits, A.J.; Wood, D.H. The response of turbulent boundary layers to sudden perturbations. Annu. Rev. Fluid Mech. 1985, 17, 321-358. [CrossRef]

13. Smits, A.J.; Matheson, N.; Joubert, P.N. Low Reynolds number turbulent boundary layers in zero and favourable pressure gradients. J. Ship Res. 1983, 27, 147-157. [CrossRef]

14. Smits, A.J.; Joubert, P.N. Turbulent boundary layers on bodies of revolution. J. Ship Res. 1982, 26, 135-147. [CrossRef]

15. Baskaran, V.; Smits, A.J.; Joubert, P.N. A turbulent flow over a curved hill. Part 1. Growth of an internal boundary layer. J. Fluid Mech. 1987, 182, 47-83. [CrossRef]

16. Baskaran, V.; Smits, A.J.; Joubert, P.N. A turbulent flow over a curved hill. Part 2. Effects of streamline curvature and streamwise pressure gradient. J. Fluid Mech. 1991, 232, 377-402. [CrossRef]

17. Alving, A.E.; Smits, A.J.; Watmuff, J.H. Turbulent boundary layer relaxation from convex curvature. J. Fluid Mech. 1990, 211, 529-556. [CrossRef]

18. Saddoughi, S.G.; Joubert, P.N. Lateral straining of turbulent boundary layers. Part 1. Streamline divergence. J. Fluid Mech. 1991, 229, 173-204. [CrossRef]

19. Panchapakesan, N.R.; Nickels, T.B.; Joubert, P.N.; Smits, A.J. Lateral straining of turbulent boundary layers. Part 2. Streamline convergence. J. Fluid Mech. 1997, 349, 1-30. [CrossRef]

20. Cheng, H.; Castro, I.P. Near-wall flow development after a step change in surface roughness. Bound. Layer Meteorol. 2002, 105, 411-432. [CrossRef]

21. Chamorro, L.P.; Porté-Agel, F. Velocity and surface shear stress distributions behind a rough-to-smooth surface transition: A simple new model. Bound. Layer Meteorol. 2009, 130, 29-41. [CrossRef]

22. Loureiro, J.B.R.; Sousa, F.B.C.C.; Zotin, J.L.Z.; Freire, A.P.S. The distribution of wall shear stress downstream of a change in roughness. Int. J. Heat Transf. Fluid Flow 2010, 31, 785-793. [CrossRef] 
23. Efros, V.; Krogstad, P. Development of a turbulent boundary layer after a step from smooth to rough surface. Exp. Fluids 2011, 51, 1563-1575. [CrossRef]

24. Harun, Z.; Monty, J.P.; Mathis, R.; Marusic, I. Pressure gradient effects on the large-scale structure of turbulent boundary layers. J. Fluid Mech. 2013, 715, 477-498. [CrossRef]

25. Hanson, R.E.; Ganapathisubramani, B. Development of turbulent boundary layers past a step change in wall roughness. J. Fluid Mech. 2016, 795, 494-523. [CrossRef]

26. Li, M.; de Silva, C.M.; Rouhi, A.; Baidya, R.; Chung, D.; Marusic, I.; Hutchins, N. Recovery of wall-shear stress to equilibrium flow conditions after a rough-to-smooth step change in turbulent boundary layers. J. Fluid Mech. 2019, 872, 472-491. [CrossRef]

27. Saavedra, J.; Poggie, J.; Paniagua, G. Response of a turbulent boundary layer to rapid freestream acceleration. Phys. Fluids 2020, 32, 045105. [CrossRef]

28. Smits, A.J.; Marusic, I. Wall-bounded turbulence. Phys. Today 2013, 66, 25-30. [CrossRef]

29. Saito, N.; Pullin, D. Large eddy simulation of smooth-rough-smooth transitions in turbulent channel flows. Int. J. Heat Mass Transf. 2014, 78, 707-720. [CrossRef]

30. Antonia, R.A.; Luxton, R.E. The response of a turbulent boundary layer to a step change in surface roughness Part 1 . Smooth to rough. J. Fluid Mech. 1971, 48, 721-761. [CrossRef]

31. Antonia, R.A.; Luxton, R.E. The response of a turbulent boundary layer to a step change in surface roughness. Part 2. Rough-tosmooth. J. Fluid Mech. 1972, 53, 737-757. [CrossRef]

32. Ismail, U. Simulations of Non-Equilibrium Rough-Wall Flows. Ph.D. Thesis, Iowa State University, Ames, IA, USA, 2018.

33. Ismail, U.; Zaki, T.A.; Durbin, P.A. Simulations of rib-roughened rough-to-smooth turbulent channel flows. J. Fluid Mech. 2018, 843, 419. [CrossRef]

34. Ismail, U.; Zaki, T.A.; Durbin, P.A. The effect of cube-roughened walls on the response of rough-to-smooth (RTS) turbulent channel flows. Int. J. Heat Fluid Flow 2018, 72, 174-185. [CrossRef]

35. Van Buren, T.; Williams, O.; Smits, A.J. Turbulent boundary layer response to the introduction of stable stratification. J. Fluid Mech. 2017, 811, 569-581. [CrossRef]

36. Van Buren, T.; Hellström, L.H.O.; Smits, A.J. Turbulent pipe flow response to rough-to-smooth step change in roughness: Flow structure. In Proceedings of the Turbulence and Shear Flow Phenomena 11, Southampton, UK, 30 July-2 August 2019; University of Southampton: Southampton, UK, 2019.

37. Van Buren, T.; Floryan, D.; Ding, L.; Hellström, L.H.O.; Smits, A.J. Turbulent pipe flow response to a step change in surface roughness. J. Fluid Mech. 2020, 904, A38. [CrossRef]

38. Smits, A.J.; Ding, L.; Van Buren, T. Flow over a square bar roughness. In Proceedings of the Turbulence and Shear Flow Phenomena 11, Southampton, UK, 30 July-2 August 2019; University of Southampton: Southampton, UK, 2019.

39. Saxton-Fox, T.; Ding, L.; Smits, A.J.; Hultmark, M. Coherent structure deformation in a turbulent pipe flow with a spatiallydeveloping pressure gradient. In Proceedings of the Turbulence and Shear Flow Phenomena 11, Southampton, UK, 30 July-2 August 2019; University of Southampton: Southampton, UK, 2019.

40. Ding, L.; Smits, A.J. Relaxation of turbulent pipe flow downstream of a square bar roughness element. J. Fluid Mech. 2021. (under review)

41. Hellströ, L.H.O.; Sinha, A.; Smits, A.J. Visualizing the Very Large Scale Motions in fully developed turbulent pipe flow. Phys. Fluids 2011, 23, 011703. [CrossRef]

42. Hellström, L.H.O.; Smits, A.J. The energetic motions in turbulent pipe flow. Phys. Fluids 2014, 26, 125102. [CrossRef]

43. Goswami, S.; Hemmati, A. Evolution of turbulent pipe flow recovery over a square bar roughness element at a range of Reynolds numbers. Phys. Fluids 2021, 33, 035113. [CrossRef]

44. Ding, L.; Saxton-Fox, T.; Hultmark, M.; Smits, A.J. Effects of pressure gradient and streamline curvature on the statistics of a turbulent pipe flow. In Proceedings of the Turbulence and Shear Flow Phenomena 11, Southampton, UK, 30 July-2 August 2019; University of Southampton: Southampton, UK, 2019.

45. Lee, M.; Moser, R.D. Direct numerical simulation of turbulent channel flow up to $\operatorname{Re} \tau \approx 5200$. J. Fluid Mech. 2015, 774, 395-415. [CrossRef]

46. Bernard, P.S.; Wallace, J.M. Turbulent Flow: Analysis, Measurement, and Prediction; John Wiley \& Sons: Hoboken, NJ, USA, 2002.

47. Rotta, J.C. Statistical theory of nonhomogeneous turbulence. Z. Phys. 1951, 129, 547-572. [CrossRef]

48. Crow, S.C. Viscoelastic properties of fine-grained incompressible turbulence. J. Fluid Mech. 1968, 33, 1-13. [CrossRef] 